\title{
Comportamiento de la deuda pública del Gobierno Central de Honduras 2002-2012
}

Eric Yovanny Andino Amaya ${ }^{1}$

\section{RESUMEN}

En los últimos años, la deuda pública es una de las variables macroeconómicas de estudio y de las más críticas producto del alto endeudamiento y persistentes déficits fiscales en varios países europeos como: Grecia, España e Italia, entre otros. Adicionalmente, el cambio de forma reiterada del techo de la deuda de Estados Unidos y el elevado endeudamiento de Japón, sumado al incumplimiento del pago de la deuda en el caso de algunos países de Latinoamérica, por ejemplo Venezuela, Ecuador, Brasil, México, Costa Rica y Argentina, fueron factores que afectaron su desempeño económico.

El objetivo de esta investigación es analizar el comportamiento de la deuda pública del Gobierno Central de Honduras en el periodo de 2002-2012, a través de la explicación de la tendencia, ciclos, composición de los componentes para establecer sus características y el diagnóstico de los indicadores e índices macroeconómicos en materia fiscal con respecto al endeudamiento público del Gobierno Central.

El endeudamiento público del Gobierno Central mostró un comportamiento con altibajos en el periodo del año 2002 a 2012, inicialmente experimentó una disminución del año 2005 a 2007 producto de la condonación de la deuda pública externa; pero, en los años posteriores se incrementó. Las causas del endeudamiento varían, del año 2002 a 2007 fueron resultado del financiamiento de la inversión pública; en cambio, del año 2008 a 2012 su crecimiento fue producto especialmente del excesivo aumento del gasto corriente, sumado a la crisis financiera mundial y política interna

\footnotetext{
${ }^{1}$ Beneficiario de una beca básica de la DICYP, Departamento de Métodos Cuantitativos, Facultad de Ciencias Económicas, UNAH: eandinos@gmail.com
} 
de 2009, situaciones que modificaron su estructura al pasar el endeudamiento público interno de un $10 \%$ del total en el año 2002, a aproximadamente el $50 \%$ en el año 2012; asimismo, el endeudamiento incrementó su proporción con respecto al PIB, empeorando la situación financiera fiscal, escenarios por los cuales Standard \& Poor's y Moody's disminuyeron la calificación de riesgo en la inversión de deuda varias veces en el año 2012, situación que no favorece al fomento de la inversión y al crecimiento económico a largo plazo.

Palabras claves: endeudamiento público, deuda interna pública, deuda externa pública, déficit fiscal, presupuesto.

\section{ABSTRACT}

In recent years, public debt is one of the macroeconomic variables of the study and the most critical product of high debt and persistent fiscal deficits in several European countries like Greece, Spain and Italy, among others. Additionally, the changing shape of the US debt and high debt in Japan, coupled with the failure to pay the debt in the case of some Latin American countries, such as Venezuela, Ecuador, Brazil, Mexico, Costa Rica and Argentina, factors affecting their economic performance. The objective of this research is to analyze the behavior of the Public Debt of the Central Government of Honduras in the period 2002-2012 through an explanation of trend, cycles, and composition of components to establish its characteristics, diagnostic indicators and fiscal macroeconomic indicators regarding the public debt of the Central Government. The public debt of the Central Government showed a behavior with ups and downs in the period from 2002 to 2012, initially experiencing a decline from 2005 to 2007 result of the cancellation of the external public debt; but increasing in subsequent years. The causes of indebtedness varied, from 2002 to 2007 resulting from financing of public investment, however the growth from 2008 to 2012 was caused by the increase in current spending, coupled with the global financial crisis and domestic politics of 2009 situations that changed its structure to pass the internal public debt of $10 \%$ of the total in 2002 , to about $50 \%$ in 2012; also borrowing increased its proportion to GDP, worsening the financial situation fiscal scenarios for which Standard \& Poor's and Moody's lowered the credit rating on debt investment several times in 2012, a situation that is not conducive to the promotion of investment and economic growth in the long term.

Keywords: public debt, public domestic debt, public external debt, fiscal deficit, fiscal budget. 


\section{INTRODUCCIÓN}

La macroeconomía moderna comprende el estudio de las variables como el crecimiento económico, desempleo, inflación, saldo comercial y saldo fiscal; este último directamente relacionado con el endeudamiento público, el cual actualmente es una de las variables económicas crítica del país, por esta razón, la presente investigación tiene como objetivo analizar el comportamiento de la deuda pública del Gobierno Central de Honduras (GCH) durante el periodo del año 2002 al 2012.

En esta investigación inicialmente se realizó un análisis del presupuesto del Gobierno Central de Honduras con el objetivo de determinar tendencia, estructura, ciclos y singularidades de cada uno de sus componentes; de igual forma se efectuó un estudio del comportamiento del endeudamiento público y, finalmente, un análisis comparativo de índices e indicadores fiscales de Honduras y los países centroamericanos, con el propósito de generar información técnica que describa características que pueden ser útiles en la elaboración de políticas económicas orientadas a la sostenibilidad del endeudamiento público u otras medidas en materia de políticas económica, esencialmente en el área fiscal o de base para futuros estudios de tipo correlacional o causal.

\section{METODOLOGÍA}

El enfoque de la investigación es cuantitativo, debido a que se realizó la medición del comportamiento de la deuda pública en un periodo de tiempo. La recolección de los datos e información se fundamentó en la medición, es decir, valores numéricos que se analizaron por medio de herramientas estadísticas con el objetivo de aportar evidencia con respecto a los lineamientos de investigación.

Los datos fueron obtenidos a través de informes generados por los entes encargados en materia fiscal. Adicionalmente, se aplicó una lógica deductiva al pasar de lo general a lo particular. El diseño es una investigación no experimental, porque no existe manipulación de las variables independientes, sino observar el fenómeno en el contexto natural y por su dimensión temporal será de corte transversal, debido a que se realizó una recolección única de datos. 
El alcance de la investigación es descriptiva debido a que el objetivo fue describir el comportamiento, estructura y característica de la deuda pública del GCH en el tiempo de estudio, así como realizar comparaciones entre variables macroeconómicas en materia fiscal a través de la recolección, medición y evaluación de las diferentes variables, aspectos y dimensiones del fenómeno, que dieran respuesta a las siguientes preguntas en el periodo de estudio del año 2002 al 2012: ¿Cuál es la estructura y tendencia de los componentes del presupuesto? ¿Cuál es el comportamiento y composición de la deuda pública? ¿Cuál es la tendencia de la deuda pública interna y externa? ¿Cuál es la situación financiera en materia fiscal? El valor de esta investigación es mostrar los ángulos y las dimensiones del comportamiento del endeudamiento público durante el periodo 2002-2012.

\section{Presupuesto del Gobierno Central de Honduras, 2002-2012}

El presupuesto, según la Secretaría de Finanzas (SEFIN), está conformado por el ingreso total y el gasto total; el primero compuesto por el ingreso corriente, ingreso de capital y fuentes de financiamiento; el segundo por el gasto corriente, gasto de capital y las aplicaciones financieras. A continuación se presenta un análisis de cada uno de los componentes.

Ingreso total

Gráfico 1. Ingreso total del Gobierno Central de Honduras, 2002 - 2012

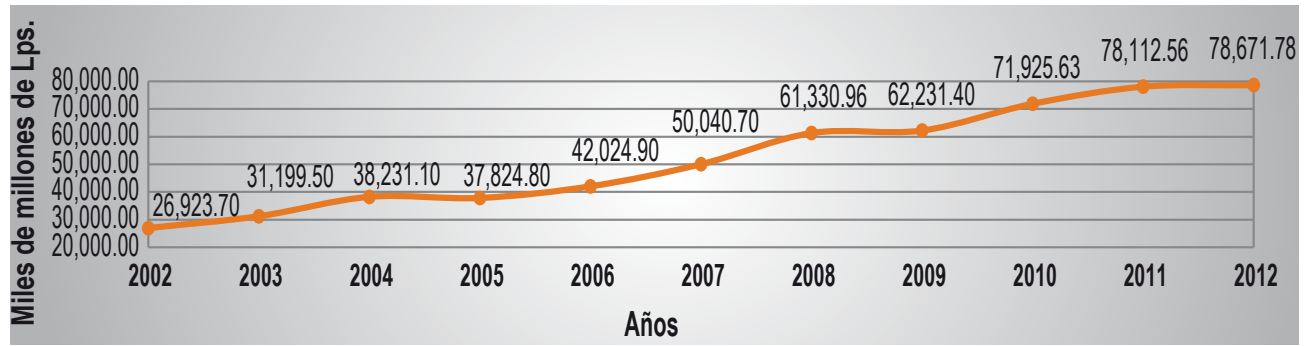

Fuente: Memorias de la Secretaría de Finanzas Públicas de Honduras (SEFIN), 2002-2012.

El ingreso total en el período mostró una tendencia positiva, con un valor promedio anual de L. 52,592.46 millones, su tasa de crecimiento anual promedio fue del $11.18 \%$, aumentó 2.92 veces, sus mayores crecimientos anuales se dieron a inicio del periodo entendido entre los años 2002 al 2008, con una tasa promedio anual del $13.8 \%$, en cambio, en los últimos años (2009-2012) mostró un desaceleramiento, reduciéndose alrededor de la mitad $(6.59 \%)$ con un comportamiento irregular. 
El crecimiento del ingreso total en el periodo fue resultado principalmente del aumento del ingreso corriente, el cual disminuyó su aportación $(78.99 \%$ de $2002-$ 2008; $56.44 \%$ de 2009 -2012), resultado de la reducción de la actividad económica desde el 2008, producto de la crisis financiera mundial sumado a la crisis política interna de 2009. En segundo lugar, el componente más influyente fueron las fuentes de financiamiento (18.32\% de 2002-2008; $39.71 \%$ de 2009-2012), el cual manifestó un mayor dinamismo por el incremento de los déficit fiscales y el endeudamiento público a partir de 2008 . Finalmente, el $74.16 \%$ del crecimiento del ingreso total en el tiempo de estudio, fue resultado de las variaciones del ingreso corriente, el 22.91 $\%$ a las fuentes de financiamiento y el restante $2.93 \%$ al ingreso de capital. Con respecto a su estructura el ingreso total durante el periodo de análisis está conformado principalmente por el ingreso corriente $(72.31 \%)$, fuente de financiamiento $(21.50 \%)$ e ingreso de capital $(6.19 \%)$.

\section{Ingreso corriente}

El promedio anual fue de L. 38,031.10 millones, con una tendencia general creciente en el periodo, aumentando 3.18 veces, sus mayores variaciones porcentuales anuales se dieron en los años de 2002 a 2008, con una tasa promedio anual de $14.47 \%$, en comparación a los últimos cuatros años del periodo (5.76 \%). La tasa de crecimiento anual promedio fue del $11.30 \%$ en el periodo, el $92.30 \%$ del incremento fue producto de los cambios del ingreso tributario, seguido por el $6.54 \%$ producto del ingreso no tributario; ambos componentes causaron el $98.84 \%$ de las variaciones porcentuales del total. Es fundamental mencionar que el ingreso corriente alcanzó crecimientos superiores al $13 \%$ entre los años 2004 a 2006, cuando la tasa de crecimiento económica fue del $6 \%$, demostrando una relación directa con los ciclos económicos y la volatilidad de la recaudación tributaria hondureña.

El ingreso corriente es el componente más importante en la conformación del ingreso total en el periodo (72.31\%), igualmente el ingreso tributario en la composición del ingreso corriente $(91.12 \%)$, por esta razón es necesario realizar un análisis del ingreso tributario con el objetivo de entender el comportamiento del ingreso total, el cual representó el $66.23 \%$.

\section{Ingreso tributario}

El promedio fue de L. 34, 653.07 millones, mostró una tendencia creciente, aumentando 3 veces, de igual forma que los ingresos anteriores, sus mayores variaciones anuales se dieron en los años de 2002-2008, con una tasa promedio anual del 
$13.68 \%$, aproximadamente el doble comparada con los últimos cuatro años del periodo ( $7.67 \%$ ), la tasa de crecimiento promedio anual fue del $11.50 \%$ en el periodo. El $98.35 \%$, de su crecimiento se debió a los impuesto sobre la producción, consumo y venta (41.10\%), impuesto sobre la renta (37.26 \%), e impuestos sobre servicios y actividades específicas (19.99\%), reafirmando la dependencia de la actividad económica.

\section{Ingreso de capital}

El promedio en el periodo fue de L. 3,255.90 millones, con una tendencia creciente, pero más leve en comparación con las anteriores; con un aumento de 1.29 veces, su tasa anual promedio fue de $10.04 \%$, no muy representativa debido a la alta dispersión de los datos. Su mayor descenso se dio en el año 2003 (-50.06 \%), resultado de la disminución de las transferencias y donaciones de capital, provocando el $99.56 \%$ de sus variaciones.

El ingreso de capital se redujo en los años anteriores a la condonación de la deuda pública externa, de 2005 a 2009, su tendencia fue positiva, pero con cambios anómalos, con un crecimiento promedio anual del $28.25 \%$, causado en su totalidad por las donaciones y transferencia de capital; posteriormente en los años 2010 y 2011, el ingreso de capital mostró un comportamiento negativo (-9.67 \% y $-14.99 \%$, respectivamente), resultado en un $95 \%$ de las variaciones de las transferencia y donaciones de capital. En el año 2012 se recuperó al aumentar el $8.02 \%$ como consecuencia de las transferencia y donaciones de capital. En definitiva, el $106.58 \%$ del crecimiento del ingreso de capital en el periodo de análisis fueron causadas por los cambios de las transferencia y donaciones de capital. Con respecto a su estructura, está conformada principalmente por las transferencias y donaciones de capital (97.29\%), las que contribuyeron con más del $87 \%$ anual.

\section{Fuentes de financiamiento}

El promedio en el tiempo de estudio fue de L.11, 305.46 millones, con una tendencia creciente, aumentando 4.44 veces, siendo el valor más alto de los componentes del ingreso total; asimismo, mostró el comportamiento más irregular. Las fuentes de financiamientos se contrajeron en los dos primeros años del periodo de la condonación de la deuda pública externa (2005- 2007), sobresaliendo el años 2005, el cual provocó que el ingreso total disminuyera en un $1.06 \%$, debido a su elevada contracción (-43.47\%), a diferencia del año 2008, en el cual las fuentes de financiamiento alcanzaron su más alto crecimiento (93.99\%), causando a su vez el mayor 
incremento del ingreso total $(22.56 \%)$ en el periodo; igualmente, mostró tasas de crecimiento altas en los años 2003 (85.24\%) y el 2010 (53.13\%); en los dos últimos años del tiempo de estudio, decreció $0.27 \%$ y $16.26 \%$, respectivamente.

Las fuentes de financiamiento produjeron más del $10 \%$ del crecimiento anual del ingreso total en los años 2003, 2004, 2008 y 2010, producto de su elevado crecimiento relacionado directamente con los déficits fiscales. La tasa de crecimiento promedio anual fue del $19.34 \%$ en el periodo, el $57.70 \%$ del crecimiento fue producto de las variaciones del endeudamiento público más el $39.67 \%$ de la obtención de préstamos; ambos componentes conforman el $97.36 \%$ del total.

Gasto total

Gráfico 2. Gasto total del Gobierno Central de Honduras, 2002-2012

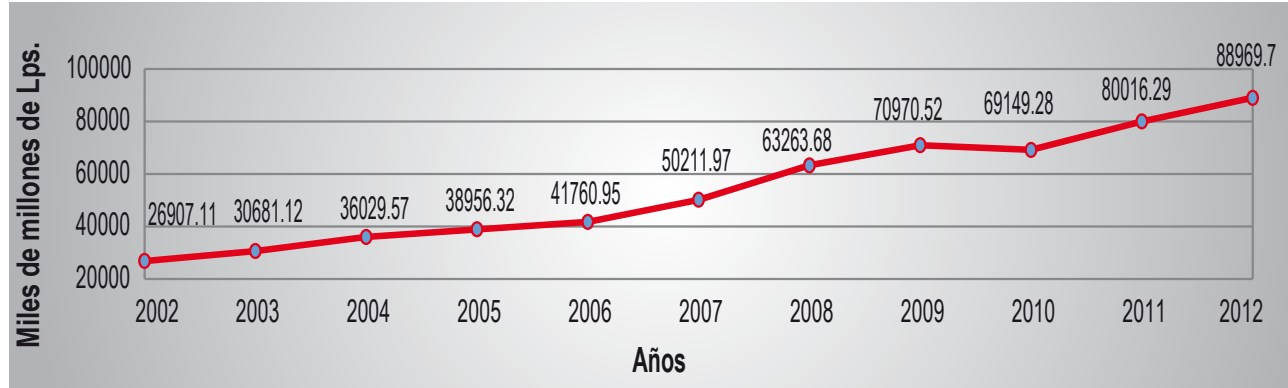

Fuente: Memorias de la Secretaría de Finanzas Públicas de Honduras (SEFIN), 2002-2012.

El gasto total promedio fue de L. 54,265.14 millones en el periodo, la tendencia general mostró un comportamiento positivo, con una tasa promedio anual de $12.03 \%$, aumentando 3.31 veces, sus mayores incrementos anuales fueron en los primeros años (2002 a 2008), con una tasa media anual del $13.69 \%$, mayor en $4.56 \%$ a sus últimos años (2009-2012), es importante mencionar que las tasas mostraron una alta dispersión.

Las variaciones del gasto total fueron resultado del incremento del gasto corriente, el cual disminuyó su contribución en los últimos años, como consecuencia de la reducción del ingreso (86.40 \% de 2002 a 2008; $64.87 \%$ de 2009 a 2012). En segundo lugar, el componente más influyente fue el gasto de capital $(7.89 \%$ de 2002 a 2008; 17.33 \% de 2009 a 2012). Es importante mencionar que las aplicaciones financieras incrementaron su participación en los últimos años debido a la crisis económica mundial y política interna del país en 2009 , las que incrementaron el déficit fiscal. 
En definitiva, el $80.47 \%$ del crecimiento del gasto total en el periodo se debió principalmente a variaciones del gasto corriente, el $10.47 \%$ al gasto de capital y $9.06 \%$ a las aplicaciones financieras; asimismo, su estructura está conformada por el gasto corriente $(73.26 \%)$, el gasto de capital (18.77\%) y las aplicaciones financieras (7.97 $\%)$, lo anterior evidencia la importancia del gasto corriente.

\section{Gasto corriente}

El promedio anual fue de L. 40,171.79 millones, con una tendencia creciente en el periodo, aumentando 3.45 veces, sus mayores variaciones porcentuales anuales se dieron en los años de 2002 a 2008 con una tasa media anual de $16.56 \%$, en los últimos cuatros años disminuyó a $7.80 \%$. La tasa anual promedio fue de $13.38 \%$, su crecimiento fue resultado del gasto de consumo (68.54\%), trasferencias corrientes (15.94 \%), la asignación global para erogaciones corrientes (8.42\%) y renta de la propiedad $(8.20 \%)$.

La tasa de crecimiento menor del gasto corriente en el periodo fue en el año 2010 (6.65 \%), resultado de la leve reducción del gasto de consumo $(-0.33 \%)$, siendo la única tasa de decrecimiento en el periodo; en el resto de los años las tasas de crecimiento fueron superiores a $6.90 \%$, sobresaliendo los años $2007(24.57 \%)$ y 2008 (24.05\%), producto principalmente del crecimiento del gasto de consumo que estimuló el incremento anual del gasto corriente en aproximadamente el $60 \%$.

\section{Gasto de consumo}

Fue uno de los componentes más importantes en la conformación del gasto total $(50.92 \%)$, sumado a las transferencias corrientes (13.45\%), ambos conformaban el $64.37 \%$ del total en el periodo; también en la estructura del gasto corriente, en donde el gasto de consumo (68.71\%) y las transferencias corrientes (18.93\%) representaban el $87.63 \%$ del total; ambos componentes determinaron cerca del $80 \%$ de las variaciones del gasto corriente.

El gasto de consumo promedio anual en el periodo fue de L. 27,629.94 millones, aumentando 3.35 veces, su tasa de crecimiento media fue del $13.40 \%$, alrededor del $80 \%$ de sus cambios corresponden a sueldos y cargas sociales, su comportamiento fue muy parecido al gasto corriente. Con respecto a las transferencias corrientes, la media anual fue de L. 7,300.16 millones, aumentando de 2.88 veces, su tasa de crecimiento promedio fue del $12.72 \%$, con el comportamiento más irregular, su 
menor tasa de crecimiento anual fue en el año 2009 (-23.31\%) y su mayor corresponde al $2008(52.28 \%)$.

\section{Gasto de capital}

El promedio en el periodo fue de L.10, 120.14 millones, con una tendencia creciente, aumentando 2.72 veces, su tasa anual promedio fue de $10.90 \%$, no muy representativa debido a la variación de los datos. Sus menores tasas de crecimiento fueron en el año $2006(-27.16 \%)$ resultado en un $96.28 \%$ debido a la disminución de la inversión real directa de capital (IRDC); seguido por el año 2010 (-24.59 \%), ocasionado de igual forma en un $60.65 \%$ por la reducción IRDC.

En cambio, los años de mayor crecimiento fueron el 2008 (76.30\%), procedente de los acelerados aumentos de las transferencias de capital (46.41\%) sumado a la IRDC (43.37\%), ambos componentes causaron el $89.78 \%$ de su variación; el año 2011 se destacó como el segundo crecimiento más elevado (42.96 \%) producto del crecimiento de la IRDC (28.54 \%), transferencia de capital (38.04 \%) y la inversión financiera (33.66 \%).

El crecimiento promedio de 2002 a 2008 fue del $11.24 \%$, levemente mayor (0.95\%) comparado con los últimos años. El $67.50 \%$ del crecimiento del gasto de capital fue causado por la IRDC, seguido por las transferencias de capital (25.63\%); ambos constituyen el $93.13 \%$ de la variación total del periodo. El gasto de capital estaba conformado especialmente por la IRDC $(47.73 \%)$ y las transferencias de capital (43.59\%); ambos componentes constituían el $91.32 \%$ del total. El gasto de capital es el componente más importante del gasto desde el punto de vista de la política fiscal, debido a que promueven el crecimiento sostenible a L/P.

\section{Aplicaciones financieras}

El promedio en el tiempo analizado fue de L.3,973.21 millones, con una tendencia creciente, aumentando 3.81 veces, sus mayores crecimientos anuales se dieron en los años 2009 (114.41\%), 2003 (93.05\%) y 2011 (50.51\%); el $71.15 \%$ de la variación anual en el 2009 se debió al aumento de la disminución de pasivos; en cambio, en el 2003, el $73.96 \%$ fue resultado de la inversión financiera y en el año 2011 el $86.93 \%$ fue producto del aumento de la amortización de la deuda interna a L/P.

Contrario a lo anterior, las aplicaciones financieras mostraron decrecimiento en varios años, entre los que se destacan: 2005 (-27.78 \%), 2008 (-45.32 \%) y $2010(-35.31 \%)$; 
la reducción de 2005 se debió principalmente a la disminución de la amortización de la deuda interna por títulos valores a corto plazo que provocó el $82.64 \%$ de la variación. En el 2008 fue resultado de la amortización de la deuda interna a L/P (96 \%) y en el 2010 se debió en su totalidad a una reducción de otros pasivos, lo cual solo formó parte de las aplicaciones financieras en seis años del periodo y desapareció desde 2010.

El crecimiento promedio anual de las aplicaciones financieras fue de $13.21 \%$ del año 2002 a 2008 y $42.71 \%$ de 2009 a 2012, incentivado por el incremento de los déficit fiscales que aumentó el endeudamiento público. La tasa promedio anual fue de 24.31 $\%$, la más alta de los componente del gasto total; asimismo mostró uno de los comportamiento más irregulares.

Los cambios porcentuales de las aplicaciones financieras fueron causadas por la amortización de la deuda externa a L/P (29.09 \%), amortización de la deuda interna por títulos valores a corto plazo (22.54 \%) y amortización deuda interna a L/P (19.74 $\%)$; estos tres componentes representaron el $71.41 \%$ del total. Con relación a su estructura, estaba conformada principalmente por amortización de la deuda externa a L/P (49.73\%), amortización de la deuda interna a L/P y endeudamiento público $(33.65 \%)$ y amortización de la deuda interna por títulos valores a corto plazo (7.87 $\%$ ); estos tres representaban el $91.25 \%$ del total.

\section{Déficit fiscal del Gobierno Central de Honduras 2002-2012}

Gráfico 3. Ingreso y gasto del Gobierno Central de Honduras, 2002 - 2012

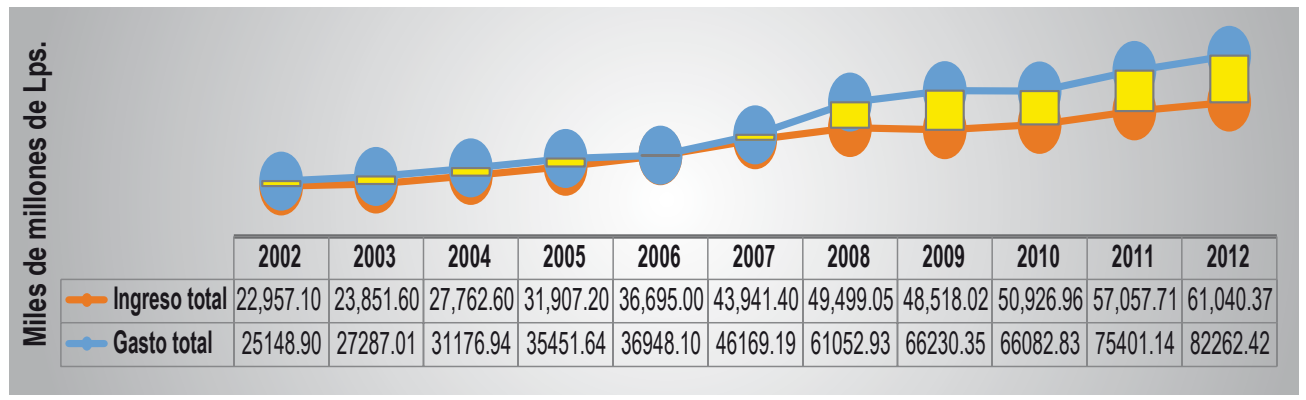

Fuente: Memorias de la Secretaría de Finanzas Públicas de Honduras (SEFIN), 2002-2012.

Ingreso del Gobierno Central

El promedio del ingreso anual (ingreso corriente agregado al capital) en el periodo fue de L. 41,287.00 millones, con un crecimiento promedio anual del $10.79 \%$, 
aumentando 2.66 veces, sus mayores tasas de crecimiento anuales se dieron en los años 2002 a 2008 (13.81\%); en comparación a sus últimos cuatro años, la cual se redujo a más de la mitad (5.50\%).

El $96.11 \%$ de las variaciones porcentuales anuales del ingreso fue resultado de los cambios del ingreso corriente y el resto al ingreso de capital. El ingreso esencialmente estaba conformado por el ingreso corriente (92.11\%), oscilando entre el $85.52 \%$ y el $94.50 \%$; asimismo, mostró una tendencia progresiva, resultado de su elevada tasa de crecimiento promedio anual (11.30\%), levemente superior a la del ingreso de capital, la cual se desaceleró en los últimos años debido a la disminución de la actividad económica, resultado de la crisis financiera mundial y de la crisis política interna de 2009.

\section{Gasto del Gobierno Central}

El gasto promedio anual (gasto corriente más gasto de capital) fue de L. 50,291.95 millones, con un crecimiento promedio anual del $11.98 \%$, aumentando 3.26 veces; al igual que el gasto total, sus mayores tasas de crecimiento anual se dieron en los años de 2002 a 2008 (14.34 \%), en comparación a los últimos cuatro años, en donde su crecimiento promedio disminuyó aproximadamente a la mitad (7.86 \%). Las variaciones porcentuales anuales del gasto en el periodo se debieron a los cambios del gasto corriente $(88.47 \%)$ y al gasto de capital $(11.53 \%)$.

El gasto corriente alcanzó crecimientos superiores al $11 \%$ entre los años 2002 a 2008, después de 2009 su crecimiento fue menor al $9 \%$ debido a la reducción del crecimiento de la recaudación tributaria, situación que redujo la contribución del gasto corriente en el crecimiento del gasto (91.33 \% de 2002 a 2008; $79.35 \%$ de 2009 a 2012). El gasto corriente mostró un crecimiento promedio anual superior en $2.48 \%$ al gasto de capital en el tiempo analizado.

\section{Déficit fiscal del Gobierno Central}

Los resultados fiscales en el periodo fueron deficitarios debido al crecimiento mayor del gasto con respecto al ingreso, fueron superiores en un $1.19 \%$; el déficit promedio anual fue de L. 9,004.95 millones, creció 9.68 veces en el periodo, mostrando dos comportamientos:

1. Del año 2002 a 2006, los déficits mostraron un tendencia a disminuir, su tasa de variación anual promedio fue de $-16.67 \%$, los años que más se contrajeron fueron el $2002(-50.40 \%)$ y el 2006 (-92.86 \%), siendo este último el de menor déficit en 
el periodo (L. 253.10 millones), resultado del incremento de la actividad económica que impulsó la recaudación tributaria. La tasa de crecimiento de este año es la más alta del periodo, sumada a la condonación de la deuda externa pública del Gobierno Central otorgada por los mecanismos HIPC, Club de París y MDRI.

2. De los años 2007 a 2012, los déficits públicos crecieron de forma exponencial, con una tasa de crecimiento promedio anual de $212.40 \%$. Los años con mayores variaciones porcentuales fueron 2007 (780.19\%) y 2008 (418.62 \%), resultado del crecimiento acelerado del gasto $(28.60 \%)$ con relación al ingreso $(16.20 \%)$ producto de la gestión administrativa, corrupción, reducción de la actividad económica mundial generada por la crisis financiera de Estados Unidos en el 2008, afectando la economía hondureña por su dependencia con este nación. En el año 2009, el déficit fiscal mostró un incremento del $53.30 \%$, menor que los años anteriores, causado por los factores inicialmente citados, además de la crisis política interna del país. En el año 2010, el déficit fiscal se contrajo en un $14.43 \%$ producto del incremento anual del ingreso $(4.97 \%)$ y la reducción del gasto en $(0.22 \%)$. Los siguientes años mostraron un incremento moderado (18.36 \%) en comparación con los años anteriores. El $76.23 \%$ del déficit fiscal en el periodo fue causado por el saldo deficitario de la cuenta de capital y el $23.77 \%$ corresponde al saldo deficitario de la cuenta corriente.

Considerando el origen del déficit fiscal con respecto a las cuentas, el periodo se divide en dos etapas:

1. Del año 2002 a 2007, cuyo déficit promedio anual fue de L. 2,511.15 millones, su totalidad fue resultado del saldo deficitario de la cuenta de capital fiscal; en cambio, la cuenta corriente fiscal disminuyó los déficits fiscales en L.1,869.66 millones en promedio al año; al financiarla, esta situación promueve el crecimiento económico a largo plazo, debido a que el endeudamiento es producto de la inversión pública.

2. Del año 2008 a 2012, en el cual el déficit fiscal promedio anual fue de L. 16,797.51 millones, 6.69 veces mayor que el promedio de los cinco primeros años, el 58.61 $\%$ es resultado del saldo deficitario de la cuenta de capital fiscal y el $41.39 \%$ es producto del saldo de cuenta corriente fiscal, estructura que no favorece la posición económica del Gobierno. Desde la perspectiva macroeconómica, es más admisible que el déficit fiscal sea producto de la cuenta de capital fiscal, debido a que son menores los impactos negativos en la economía, siempre que se encuentre dentro del rango aceptable, con el objetivo de promover el crecimiento sostenido a largo plazo. 


\section{Deuda pública del Gobierno Central de Honduras, 2002-2012}

Gráfico 4. Deuda pública anual del Gobierno Central de Honduras, 2002-2012

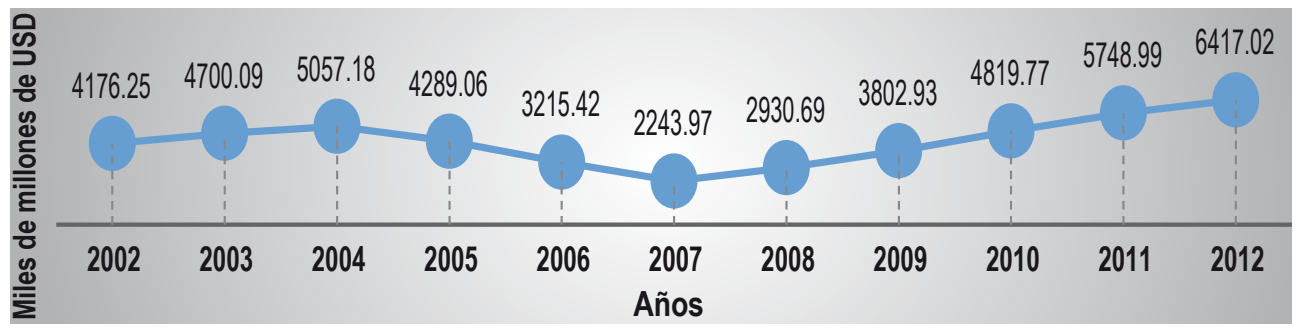

Fuente: Dirección de Deuda Pública de la Secretaría de Finanzas Públicas de Honduras (SEFIN), 2014.

El comportamiento de la deuda pública fue irregular, pero con una tendencia general creciente, principalmente en los últimos cinco años. El valor promedio fue de USD 4,309.22 millones, su valor mínimo fue en el año 2007 y el máximo corresponde a 2012 , la tasa de crecimiento promedio anual fue del $6.95 \%$ en el periodo, con oscilaciones entre $-30.21 \%$ (2007) y el $30.60 \%$ (2008).

Con respecto a su estructura, está conformada principalmente por la deuda pública externa, la cual representó tres cuartas partes del total $(75.70 \%)$, su contribución fluctuaba entre el $53.31 \%$ y el $92.09 \%$. Es importante mencionar que su participación disminuyó con el pasar de los años. En el año 2002, la deuda pública externa representaba aproximadamente el 90 \%, para el 2012 representaba cerca de la mitad, producto del aumento acelerado de la deuda interna, especialmente entre los años de 2008 a 2011, en donde la tasa promedio anual fue del $59.54 \%$, destacándose el año 2009 porque el endeudamiento público interno se duplicó (106.57\%), producto del financiamiento del déficit fiscal con endeudamiento interno por el cierre del acceso al crédito externo por la crisis política interna, sumado a la disminución de la recaudación tributaria y por la recesión económica.

Acerca de la deuda interna pública, esta representaba cerca de la cuarta parte (22.99 $\%$ ) de la deuda total, su contribución varía entre el $8.20 \%$ y el $46.14 \%$, aumentando su participación alrededor de cinco veces; el crecimiento promedio anual en el periodo fue del $25.91 \%$, casi tres veces mayor que la deuda externa pública (8.96 \%), situación que explica el cambio en la estructura. Los ciclos del endeudamiento público del GCH se dividen en tres etapas:

1. I trimestre del año 2002 al IV trimestre de 2004: en estos años el endeudamiento público mostró un leve comportamiento creciente, la tasa promedio trimestral fue del $2.36 \%$ y la anual del $9.63 \%$, aproximadamente del $87.17 \%$ de las variaciones anuales de la deuda pública total fueron causadas por cambios en la deuda externa. 
2. I trimestre de 2005 al IV trimestre de 2007: mostró un comportamiento decreciente debido a la reducción de la deuda externa pública resultado del alivio otorgado por los mecanismos HIPC, Club de París y MDRI, con el objetivo de liberar recursos públicos para combatir la pobreza, disminución del gasto por concepto de pago de intereses, sumada a la buena gestión administrativa; la tasa de variación promedio trimestral fue de $-5.7 \%$ y la anual de $-23.48 \%$. El $100.57 \%$ de las variaciones de la deuda pública total son causadas por cambios en la deuda externa pública.

3. I trimestre de 2008 al IV trimestre de 2012: con el crecimiento más elevado, la tasa promedio de la deuda pública trimestral fue del $5.52 \%$ y la anual del $23.60 \%$; ambas tasas duplican el valor de las dos etapas anteriores, sobresaliendo los años 2008 (30.60 \%), 2009 (29.76 \%) y 2010 (26.74 \%) como los años de crecimiento más acelerado producto de la gestión de los recursos administrativos (elevado gasto público), la recesión económica de 2009, la crisis política interna, la crisis financiera mundial y el bajo crecimiento económico en los años posteriores. En esta etapa el $59.68 \%$ de las variaciones fueron producidas por cambios en la deuda interna pública. Finalmente, el $97.55 \%$ de las variaciones promedio de la deuda pública del periodo fue causada por la deuda interna pública, resultado del crecimiento acelerado en los últimos cinco años (49.26\%), sumado a la disminución de la deuda externa pública del año 2005 al 2007 (-26.41 \%).

\section{Deuda externa pública del Gobierno Central de Honduras, 2002-2012}

Gráfico 5. Comportamiento de la deuda externa pública anual del Gobierno Central, 2002-2012

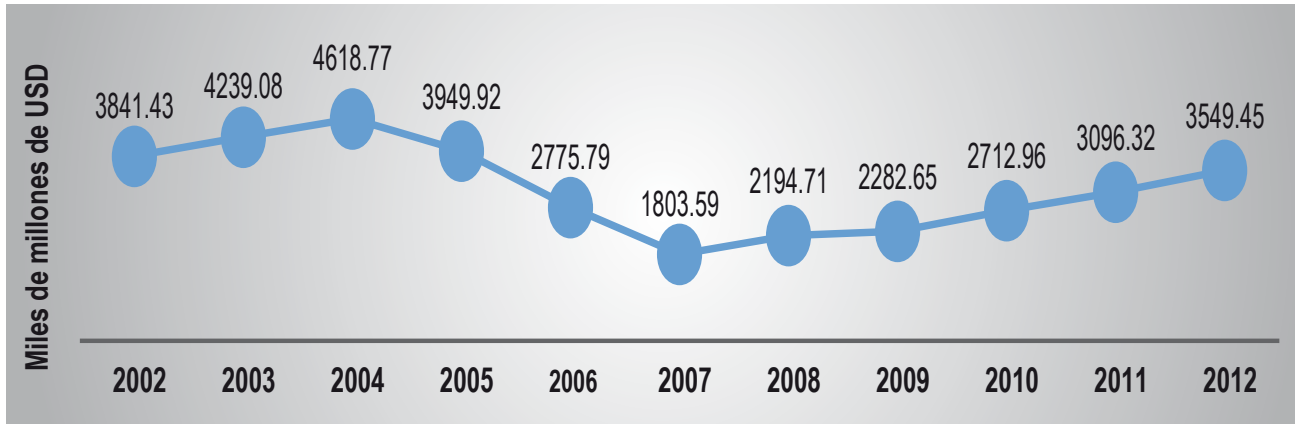

Fuente: Dirección de Deuda Pública de la Secretaría de Finanzas Públicas de Honduras (SEFIN), 2014.

La deuda externa pública total tiene un comportamiento muy parecido a la total, debido a que representa en promedio la mayor porción $(75.40 \%)$, el valor promedio anual fue de 3,187.70 millones, con un valor mínimo en el año 2007 y un máximo en el 2012. Su tendencia general es decreciente y se divide en tres fases: 
1. De 2002 a 2004 , con una tasa de crecimiento anual del $9.17 \%$, aumentando 1.2 veces.

2. Del año 2005 a 2007 , con una tasa de decrecimiento del $26.41 \%$, disminuyendo 2.20 veces, producto de su condonación.

3. Del año 2008 a 2012, con una tasa de crecimiento anual del $14.66 \%$ superior a los primeros años del periodo. La tasa promedio anual de la deuda externa pública en el periodo fue del $1.96 \%$ (creciendo 1.62 veces), no muy representativa debido a su variación irregular, resultado principalmente de la gestión administrativa del Gobierno.

\section{Deuda interna pública del Gobierno Central de Honduras, 2002-2012}

Gráfico 6. Comportamiento de la deuda interna pública anual del Gobierno Central, 2002-2012

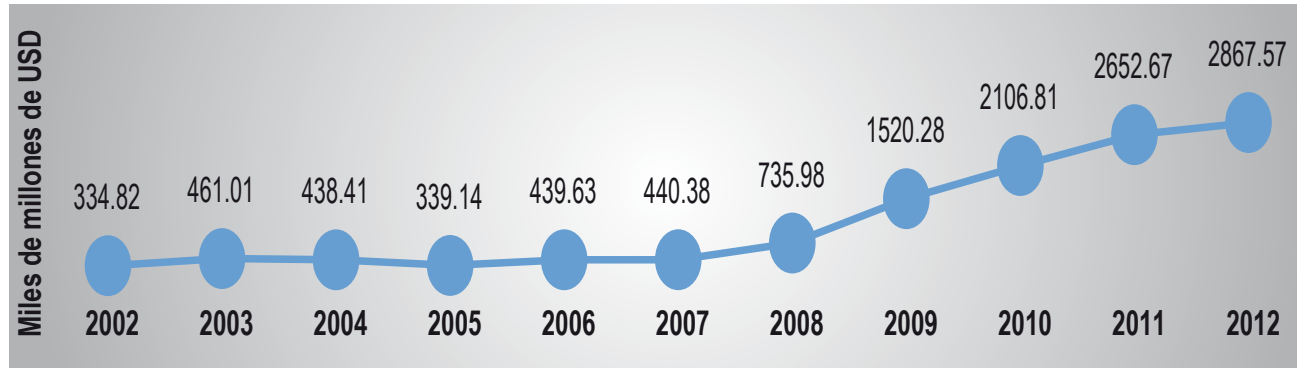

Fuente: Dirección de Deuda Pública de la Secretaría de Finanzas Públicas de Honduras (SEFIN), 2014.

La deuda pública interna promedio anual en el periodo fue de USD 1,121.52 millones, con un valor mínimo en el año 2002 y máximo en el 2012, el valor total se incrementó en 7.56 veces en el periodo, con una tasa de crecimiento anual promedio de $27.42 \%, 13.97$ veces mayor que la deuda externa pública y 3.95 veces con respecto a la total.

La tendencia general es creciente, la cual se divide en dos fases: la primera va del año 2002 a 2007, con una tasa de crecimiento promedio anual de $9.23 \%, 5.34$ veces menor comparada con el $49.26 \%$ del año 2008 a 2012, que corresponde a la segunda fase. Las tasas de crecimiento más altas corresponden a los años 2008 (67.12 \%) y el 2009 (106.57 \%),provocando cambios en su participación, para el año 2012 representaba cerca del $50 \%$, resultado de su crecimiento acelerado a partir de 2007 al pasar de USD 440.38 millones a USD 2,867.57; un incremento de 6.52 veces. 


\section{Indicadores fiscales macroeconómicos del Gobierno Central, 2002-2012}

Ingresos y gastos del Gobierno Central de los países centroamericanos

Gráfico 7. Ingreso promedio del GC \% del PIB de los países centroamericanos, 2002-2012

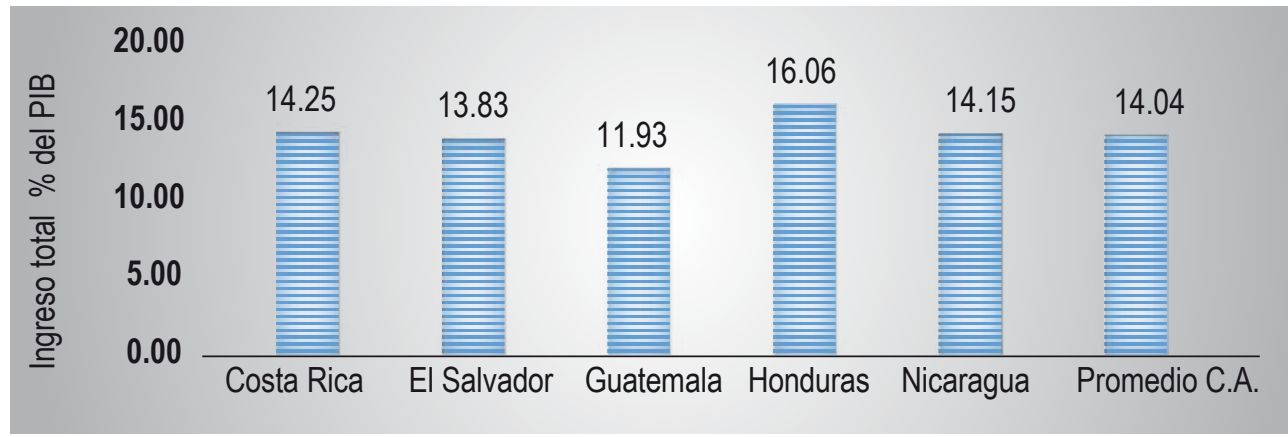

Fuente: SIMAFIR, base de datos macroeconómicos, Consejo Monetario Centroamericano, 2014.

Gráfico 8. Gasto promedio del GC \% del PIB de los países centroamericanos, 2002-2012

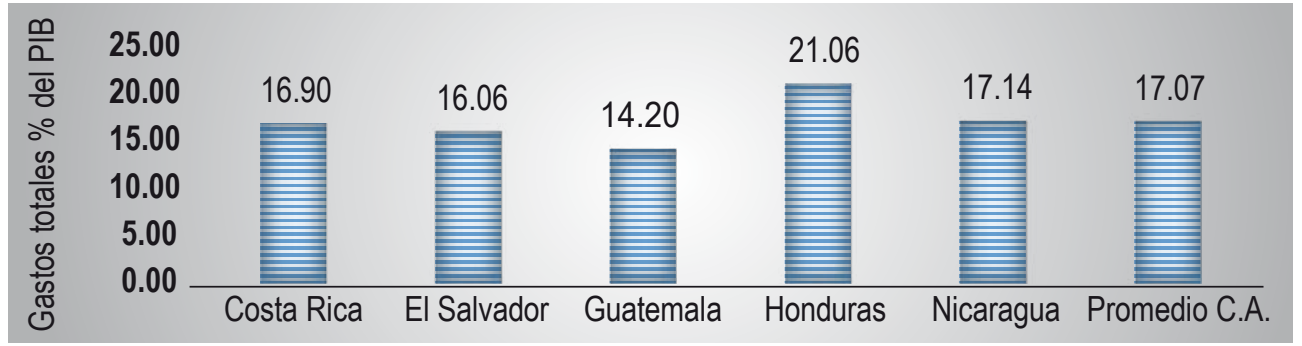

Fuente: SIMAFIR, base de datos macroeconómicos, Consejo Monetario Centroamericano, 2014.

Existe una relación directa entre los ingresos totales de los países centroamericanos y los de Honduras en el periodo de análisis. El ingreso total del GCH aumentó 2.43 veces en once años, relativamente igual al promedio de los países de Centroamérica (2.47 veces). Asimismo, el promedio anual de los ingresos totales en el periodo fue del $9.59 \%$, menor solo en $0.16 \%$ al promedio de los países de Centroamérica, debido a que el crecimiento de los ingresos superó en $1.42 \%$ del año 2003 a 2009, en los últimos tres años esta tendencia cambia, al ser menor en $3.87 \%$, lo que explica el crecimiento de la brecha.

El ingreso total promedio del GCH (USD 2,023.14 millones) es menor en USD 747.47 millones al promedio de los países de Centroamérica. Los ingresos totales del GCH 
promedio representan el $16.06 \%$ del valor de la producción de bienes y servicios finales del país en el periodo de análisis, posicionado en primer lugar con respecto a este índice, el cual fue superior en $2.02 \%$ del promedio de los países de Centroamérica, no obstante, los valores están muy por debajo del ingreso con respecto a otros países. "Los ingresos públicos forman en promedio 25 por ciento del PIB en América Latina, comparados con 42 por ciento del PIB en los países de la OCDE" (Puryear y Jewers, 2010).

Lo anterior es producto del bajo nivel de desarrollo de la región, sistemas tributarios neutros o regresivos, existencia de un gran sector informal, evasión ilegal de impuestos y las excepciones legales y vacíos en materia legal, factores que favorecen a los hogares de altos ingresos, reduciendo su carga tributaria. En América Latina, las tasas de gravámenes reglamentadas para los impuestos individuales y de sociedades sobre la renta son levemente más bajas que en otras partes del mundo, pero el impuesto sobre venta se aproxima a las normas internacionales.

En el caso de Honduras, es más dependiente de los impuestos indirectos seguidos de los impuestos directos, volviendo al sistema tributario volátil por la dependencia de la actividad económica. En términos monetarios de ingreso total y de la producción (PIB nominal USD 12,576.88 millones), ocupaba el cuarto lugar en ambos agregados económicos con respecto a los países de Centroamérica, por esta razón, al dividir el ingreso total entre el tamaño de la economía en términos corrientes, su porción fue mayor que el resto de los países.

Al igual que en el ingreso existe una relación entre los gastos totales de los países de Centroamérica y los de Honduras en el periodo, el gasto total del GCH aumentó 2.65 veces en once años, levemente superior al promedio de los países del istmo (2.48 veces). Además, el crecimiento promedio del gasto total del $\mathrm{GCH}(10.48 \%)$ fue mayor que el promedio de los países de Centroamérica en aproximadamente $1 \%$, en el periodo de 2002 a 2006 tenían una relación directa; en cambio, de 2007 a 2012 su relación fue inversa. El gasto total promedio del GCH (USD 2,684.58 millones) es menor en USD 657.05 millones al promedio de los países de Centroamérica (USD $3,341.63$ millones) en el periodo, una brecha menor comparada con el ingreso total.

La brecha entre el gasto total del GCH y el promedio de los países de Centroamérica es menor entre los años 2007 a 2009 (USD 445.53 millones), comparada con los años de 2002 a 2006 (USD 558.78 millones) producto del crecimiento acelerado del gasto del GCH, pero las diferencias se duplicaron en los últimos tres años del periodo de análisis (USD 1,032.33 millones), producto del crecimiento rápido del gasto total promedio de los países de Centroamérica, principalmente en el 2010. Las diferencias más notables fueron en el año 2007, cuando la variación del gasto total de Honduras 
(29.97 \%) fue aproximadamente el doble con respecto al promedio de los países de Centroamérica (15.23\%); diferente al año 2010, en donde la variación porcentual del GCH fue del $1.24 \%$, comparado con el $17.19 \%$ de los países de Centroamérica, alrededor de 14 veces mayor.

El gasto total promedio del GCH fue del $21.06 \%$ del valor de la producción de bienes y servicios finales del país en el periodo, obteniendo el primer lugar con relación a este índice, producto del pequeño tamaño de la economía comparada con su gasto, fue superior en $3.99 \%$ al promedio de los países de Centroamérica, es decir, el doble de la diferencia comparado con el ingreso como \% del PIB. Es importante mencionar que el gasto como \% del PIB del GCH supera en $5 \%$ los ingresos como porcentaje del PIB, situación que deteriora su posición financiera.

Este ratio en el caso de los países centroamericanos es bajo con respecto a otros países: "El gasto público nos cuenta una historia similar: en el mismo periodo alcanzó una media del $44 \%$ del PIB en los países de la OCDE, pero de solo un $25 \%$ en América Latina" (OCDE, 2008,1). El gasto de los países europeos es utilizado como una herramienta para reducir la pobreza y la desigualdad, su eficiencia se basa en proveer servicios públicos de calidad, salud y educación que mejoran la calidad del capital humano y las transferencias de fondos a las personas de bajos recursos de forma focalizada y con objetivos definidos, en los países de Latinoamérica se utiliza más como herramienta de política fiscal los impuestos.

\section{Déficits fiscales del Gobierno Central de los países centroamericanos, 2002-2012}

Gráfico 9. Déficit del GC como \% del PIB de Honduras, 2002-2012

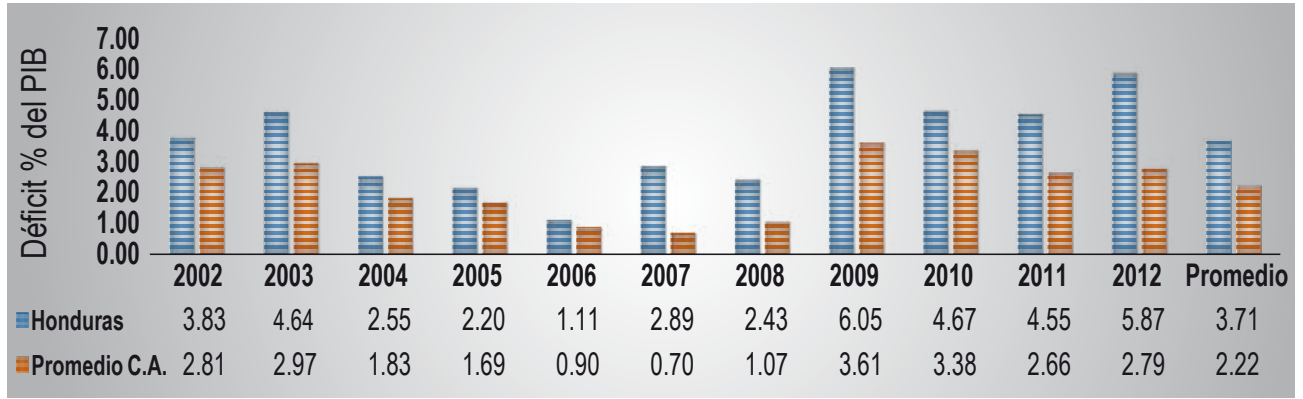

Fuente: SIMAFIR, base de datos macroeconómicos, Consejo Monetario Centroamericano, 2014.

Existe una relación directa entre el déficit fiscal promedio del GCH y de los países de C.A., en el periodo de análisis, no obstante, existen diferencias a partir de 2007, en donde las brechas son más pronunciadas. Ambos mostraron una tendencia decreciente en los años 2002 a 2006 (Honduras: -15.64 \%; países centroamerica- 
nos:-10.47\%); en este tiempo el déficit fiscal promedio anual de Honduras fue menor en USD 45.51 millones al promedio de los países de Centroamérica, resultado de que la variación media anual del ingreso superó al gasto, en el caso de Honduras en $3.62 \%$ y el promedio de los países centroamericanos en $3.10 \%$. En los siguientes años, de 2007 a 2009, el déficit fiscal promedio anual del GCH fue mayor en USD 140 millones, producto de que la variación porcentual media anual del ingreso total fue menor a la del gasto total en $3.91 \%$ en Honduras y $2.79 \%$ para el promedio de los países de Centroamérica, situación que provocó que los déficits fiscales crecieran de forma acelerada a partir de 2008. En el año 2007, el déficit fiscal del GCH se duplicó (195.04 \%) en comparación al promedio de los países centroamericanos que disminuyó en un $30.88 \%$.

En contraposición, el año 2009 muestra un crecimiento acelerado tanto del GCH (160.96\%), como del promedio de los países de Centroamérica $(261.67 \%$ ), producto principalmente de la crisis mundial y política interna del país, en los próximos años la brecha se redujo (2010: USD 209.66 millones; 2011: USD 57.84 millones). Para 2012, el déficit fiscal del GCH fue superior en USD 152.14 millones con respecto a los países centroamericanos. Finalmente, el déficit fiscal promedio del $\mathrm{GCH}$ fue levemente superior (USD 11.78 millones anuales) debido a que la tasa anual promedio del déficit fiscal del GCH (31.60\%) fue superior en $5.25 \%$ al de los países de Centroamérica, situación que deteriora la posición financiera de Honduras en comparación a los demás.

Honduras es el país con el tercer déficit fiscal del GC más alto de los países centroamericanos en el tiempo de análisis, superado por Guatemala y Costa Rica, las dos economías más grandes de Centroamérica; las cuales poseen un déficit fiscal de aproximadamente 1.5 veces mayor con respecto al de Honduras.

El Salvador, la tercera economía más grande, tiene el cuarto déficit fiscal más alto, el cual representa el $68.32 \%$ del déficit fiscal del $\mathrm{GCH}$; en último lugar se sitúa Nicaragua, con un déficit de cerca de 10 veces menor con respecto a Honduras, el déficit tiene impactos negativos debido a que tiende a elevar las tasas de interés, aumentar el endeudamiento, reducir la calificación de riesgo, incitar la inflación, reducir la inversión, entre otras.

El déficit fiscal como \% del PIB, es un índice utilizado para determinar la posición financiera del Gobierno, el valor de referencia con respecto a este índice depende de la situación económica particular desde la perspectiva económica, valores altos debilitan la posición financiera, provocando una disminución en la calificación crediticia que encarece y dificulta el acceso a la obtención de crédito por el incremento del 
riesgo e incertidumbre y disminuye la inversión extranjera directa, situaciones que afectarán la estabilidad macroeconómica, que es una condición necesaria para que la economía crezca con inflación baja.

Una consideración con respecto al tamaño del déficit fiscal como \% del PIB, es que entre más alto sea el endeudamiento público, menor debe de ser este ratio, con el objetivo de evitar problemas financieros y mejorar la de sostenibilidad de la deuda. Honduras, en términos monetarios del déficit fiscal promedio anual del GC, ocupaba el tercer lugar con respecto a los países centroamericanos, dividido el saldo entre el PIB promedio anual, el resultado es de $3.71 \%$ en el periodo de análisis, el valor más alto de los países centroamericanos, seguido por Costa Rica (2.66 \%) y en último lugar Nicaragua (0.83 \%).

La interpretación del índice es el porcentaje del déficit fiscal como una porción del ingreso anual del país, o la porción del ingreso necesaria para cubrir el déficit fiscal. Lo anterior evidencia el debilitamiento de la posición financiera del GCH en el periodo de análisis. En los países europeos, según el marco fiscal del Tratado de Maastricht, cuyo propósito es combinar la disciplina y la flexibilidad, exige a los miembros llegar a situaciones fiscales "próximas al equilibrio o con superávit" a mediano plazo y que mantengan el déficit efectivo por debajo del $3 \%$ del PIB, excepto en caso de shocks excepcionalmente fuertes.

El ratio del déficit fiscal con respecto al PIB de Honduras se contrajo en los años desde 2004 a 2006, 2008, 2010 y 2011, debido a que las variaciones porcentuales anuales del PIB nominal fueron mayores que del déficit fiscal del GCH, sobresaliendo el 2006, en donde el crecimiento del PIB nominal fue del $11.87 \%$, versus la disminución del déficit fiscal de un $55.46 \%$, dando como resultado la porción del déficit fiscal más pequeño con respecto al PIB del $1.11 \%$ en el periodo de análisis. A diferencia de los años 2007, 2009 y 2012, cuando las variaciones porcentuales anuales del PIB nominal son menores que las del déficit fiscal.

En definitiva, la tasa de crecimiento del PIB nominal de 2003 a 2012 fue del $8.91 \%$ comparada con el $31.60 \%$ del déficit fiscal, provocó el crecimiento continuo del ratio del déficit fiscal anual con respecto al PIB, principalmente en los últimos años, perjudicando la posición financiera del GC y el aumento del endeudamiento público. Según How to do a Debt Sustainability Analysis for Low-Income Countries, October (2006), del FMl y el Banco Mundial, se demuestra que un déficit fiscal de hasta 1,4 \% del PIB anual sería sostenible en condiciones normales, situación que solo se logró en el año 2006. 
Cuadro 1. PIB nominal y endeudamiento público en dólares, variaciones porcentuales y $\%$ del PIB de Honduras, $2002-2012$

\begin{tabular}{|ccccccccccccc|}
2012 & 2011 & 2010 & 2009 & 2008 & 2007 & 2006 & 2005 & 2004 & $\mathbf{2 0 0 3}$ & $\mathbf{2 0 0 2}$ & Unidades & Variables \\
\hline 18,38 & 17,67 & 15,84 & 14,58 & 13,88 & 12,36 & 10,91 & 9,76 & 8,87 & 8,23 & 7,86 & MM de dólares & PIB nominal \\
\hline 4.02 & 11.58 & 8.58 & 5.04 & 12.35 & 13.22 & 11.87 & 9.99 & 7.76 & 4.73 & 2.7 & Variación & \\
\hline 5,95 & 5,66 & 4,69 & 3,57 & 3,17 & 3,03 & 4,38 & 5,53 & 6,04 & 5,43 & 4,89 & MM de dólares & Deuda pública \\
\hline 5.11 & 20.74 & 31.25 & 12.79 & 4.43 & - & -20.90 & -8.33 & 11.09 & 11.25 & ND & Variación & total \\
\hline 1.09 & 9.16 & 22.67 & 7.75 & -7.92 & - & -32.77 & -18.32 & 3.33 & 6.52 & ND & Diferencia & \\
\hline 32.38 & 32.05 & 29.62 & 24.50 & 22.82 & 24.55 & 40.11 & 56.73 & 68.06 & 66.02 & 62.15 & Gastos totales \% del PIB \\
\hline
\end{tabular}

Fuente: SIMAFIR, base de datos macroeconómicos, Consejo Monetario Centroamericano, 2014.

\section{Deuda pública del Gobierno Central de los países centroamericano, 2002 - 2012}

El endeudamiento público de Honduras tiene un comportamiento similar al promedio de los países centroamericanos, en los años de 2003 a 2004, ambos mostraron una tendencia progresiva, ya que el crecimiento promedio anual del endeudamiento público del GCH (11.17\%) fue inferior en $1.60 \%$ con respecto al promedio de los países de Centroamérica. En los años siguientes de 2005 a 2007, ambos mostraron un decrecimiento de forma permanente, la deuda pública del GCH decreció de forma más rápida que el resto de los países centroamericanos, la tasa de variación porcentual anual promedio fue del $-20 \%$, comparada con el $-1.27 \%$ del promedio de los países de Centroamérica, debido a la condonación de la deuda externa de Honduras al entrar a la iniciativa de Países Pobres Altamente Endeudados (HIPC), situación que disminuyó la deuda pública de forma considerable; en los próximos años el endeudamiento público fue creciente, la tasa de crecimiento media anual del endeudamiento del GCH (14.86 \%) fue superior en $2.71 \%$ con relación al promedio de los países centroamericanos; en términos generales, la tasa de crecimiento media del endeudamiento público de los países de Centroamérica fue del $8.25 \%$ mayor con respecto a la de Honduras (3.67 \%) en el periodo de análisis.

El endeudamiento público total promedio de los países centroamericanos aumentó del año 2002 a 2012 en 2.14 veces, aproximadamente el doble con respecto al de Honduras (1.22 veces), la brecha del endeudamiento entre los países centroamericanos y Honduras fue de 6.31 veces en el periodo de análisis. La deuda pública total promedio anual del GCH (USD 4,759.01 millones) fue menor en USD 3,797.58 millones al promedio de los países de Centroamérica, aproximadamente la mitad (55.62\%). 
Honduras es el país con el endeudamiento público promedio más bajo en el periodo, el endeudamiento público de Costa Rica fue 2.85 veces mayor al de Honduras, el de Guatemala fue el doble y el de Nicaragua 1.25 veces mayor; situación favorable en términos nominales a Honduras.

La deuda como \% del PIB es una medida de tiempo, representa lo que necesitaría destinar de un año de producción de bienes y servicios para pagar la deuda; es necesario tomar en cuenta las limitantes del índice, entre el que se destacan el problema de etiqueta, que significa que solo se está evaluando cuánto representa la deuda de la producción del país, pero no el uso o destino de los fondos del endeudamiento, es decir, si se destina a gasto de capital o corriente, siendo la primera la mejor opción, adicionalmente el costo del financiamiento, la estructura de la deuda, el acceso a crédito, la estructura impositiva y el déficit público, son factores que el índice no toma en cuenta.

El valor más utilizado de referencia con respecto a este índice, es que la deuda pública total debe de ser menor del $50 \%$ del PIB según el Fondo Monetario Internacional. Honduras cumple con el valor de referencia en términos del promedio del periodo, el cual fue de $41.69 \%$, el segundo más bajo de los países centroamericanos, solo por arriba de Guatemala (28.45\%). La deuda pública total del GCH es superior al valor de referencia en los años comprendidos entre el 2002 a 2005, alcanzando valores cercano al $70 \%$ del PIB; los siguientes tres años (2006 al 2008) mostraron una reducción en el ratio de forma continua, muy por debajo del valor de referencia, debido a que las variaciones porcentuales promedios anuales del endeudamiento público (-19.98 \%) fueron menores a las del PIB nominal (11.69\%).

En los últimos años del periodo (2009 a 2012), el ratio se incrementó de manera acelerada debido al elevado crecimiento promedio anual del endeudamiento público (17.47 \%) con respecto al PIB (7.31\%), al pasar del $24.50 \%$ en el 2009 a $32.38 \%$ en el 2012, debilitando la sostenibilidad de la deuda pública y la posición financiera, asimismo demuestra el comportamiento despilfarrador de la administración central.

\section{CONCLUSIONES}

La deuda pública total del Gobierno Central de Honduras en el periodo de 2002 a 2012, mostró un comportamiento con altibajos, con una ligera tendencia creciente, asimismo se fraccionó en tres fases: 
1. Inicialmente decreciente ( $9.63 \%$ media anual) comprendido entre los años 2002 a 2004, el $87 \%$ de las variaciones de la deuda total se debieron a cambios de la deuda externa pública, resultado de la gestión administrativa y el bajo crecimiento económico.

2. En los siguientes años de 2005 a 2007 , se mostró un comportamiento decreciente (-23.47 \% media anual), el $100 \%$ de la reducción se debe a la deuda externa pública por el alivio otorgado por los mecanismos HIPC, Club de París y MDRI al gobierno.

3. Finalmente, de 2008 a 2012, con un crecimiento anual del $23.60 \%$, el más acelerado del periodo, alrededor del $60 \%$ de los cambios son por el endeudamiento público interno, resultado de varios factores como la crisis financiera mundial, recesión y bajo crecimiento económico del país (afecto de la recaudación tributaria), excesivo gasto y la crisis política de 2009; situación que provocó que el financiamiento de los déficit fiscales fuera endeudamiento interno por el bloqueo del sector externo y la falta de un acuerdo Stand By con el FMI. La tasa de crecimiento de la deuda pública total fue del $6.95 \%$ en el periodo.

Desde la perspectiva del tipo de financiamiento, la deuda externa pública determinó el comportamiento del endeudamiento público del Gobierno Central en los años de 2002 a 2008 y la deuda interna pública del año 2009 a 2012. La tasa de crecimiento media anual del endeudamiento público total en el periodo de análisis fue del $6.95 \%$; aproximadamente, el $97.55 \%$ de sus variaciones porcentuales promedio fueron causadas por los cambios de la deuda interna pública, producto de su crecimiento anual acelerado en los últimos cinco años (49.26\%), sumado a la reducción de la deuda externa pública del año 2005 al 2007 (-26.41\%), situaciones que cambiaron su estructura al pasar la deuda pública externa del $91.98 \%$ en el año 2002 al $44.69 \%$ en 2012 del total, en promedio la deuda externa pública representó el $75.7 \%$ del total.

Los desfases entre los ingresos y los gastos del Gobierno Central de Honduras (promedio de $1.19 \%$ ) provocaron déficits fiscales persistentes a lo largo del periodo, el crecimiento promedio anual del déficit fue aproximadamente del $100 \%(9.68$ veces), asimismo mostró dos comportamientos:

1. De 2002 a 2006, los déficit tenían un comportamiento decreciente anual promedio del $16.66 \%$, en cambio en los años restantes manifestaron un tendencia elevada al alza $(212.40 \%)$. Con respecto al origen de los déficits fiscales, fueron resultado del saldo deficitario de la cuenta de capital fiscal, financiada cierta porción por la cuenta corriente fiscal, es decir, fue producto de la inversión pública. 
2. En cambio, de 2008 a 2012, fue resultado del saldo deficitario de ambas cuentas, pero principalmente de la cuenta de capital fiscal que causó el $58.61 \%$ del desfase. Finalmente, aproximadamente el $76.23 \%$ del déficit fiscal en el periodo fue producto del déficit de la cuenta de capital, en relación al ratio del PIB solo el año 2006 mostró una posición favorecedora (1.11\% del PIB) menor al $1.4 \%$, que es el valor de referencia del país según estudios del FMI y el BM en condiciones normales. Asimismo, mostró déficits fiscales cercanos o superiores al $5 \%$ del PIB entre los años 2009 y 2012, situación que no favorece a las finanzas públicas desde el punto de vista de la sostenibilidad de la deuda pública, además en promedio los déficits fiscales anuales de Honduras, con respecto al PIB (3.72\%), fue superior al promedio de los países centroamericanos (2.22\%).

Los ingresos del Gobierno Central de Honduras como porcentaje del PIB promedio en el periodo fue del $16.06 \%$, con un comportamiento estable, el más alto con respecto a los países centroamericanos, pero debajo del promedio de América Latina (25\%) y de los países OCDE (42\%). Asimismo, son altamente dependientes de los ingresos tributarios: aproximadamente el $83.93 \%$ del ingreso total, la mayor porción del ingreso corriente son impuestos sobre la producción consumo y venta (43.13\%), más el impuesto sobre la renta $(30.14 \%)$, manifestando la volatilidad del sistema tributario además de ser más regresivo.

Con respecto al gasto total, representaban en promedio el $21.06 \%$, el más alto de los países centroamericanos, pero cercano al promedio de América Latina $(25 \%)$ y alrededor de la mitad de los países de la OCDE (44\%); el gasto de capital mostró una reducción en la composición con respecto al gasto total, al pasar de $32.26 \%$ en el años 2002 a $20.74 \%$ en el 2012, composición que no favorece a la calidad del gasto, el crecimiento económico y la sostenibilidad del endeudamiento. La deuda pública total del GC en promedio fue del $41.69 \%$ del PIB en el periodo, asimismo mostró dos comportamiento: una disminución al pasar de $62.15 \%$ del PIB en el año 2002 a $22.82 \%$ del PIB en el 2008, resultado del condonación de la deuda externa; después su proporción aumentó a 32.38 \% en el 2012, causado por el incremento del endeudamiento público interno, situación que afecta la sostenibilidad de la deuda pública y el crecimiento económico a largo plazo del país. 


\section{BIBLIOGRAFÍA}

Afonso, A. y Gaspar V. (2006). Excess Burden and the Cost of Inefficiency in Public Services Provision. ECB Working Paper Recuperado de: http:/ssrn./abstract_id-0 Baldacci, E.; Clements, C. y Gupta, S. (2003). Utilizar la política fiscal para estimular el crecimiento. Finanzas \& Desarrollo. Recuperado de: https://www.imf.org/external/pubs/ft/fandd/spa/2003/12/pdf/baldacci.pdf

Banco Central de Honduras. (2013). Programa Monetario 2013-2014. Recuperado de: http://www.bch.hn/download/programa_monetario/programa_monetario_2013_2014.pdf

Consejo Monetario Centroamericano. (20 de agosto de 2014). Base de datos macroeconómicos SIMAFIR. Recuperado de: http://www.secmca.org/simafir.html.

Daseking, C. (2002). Deuda, ¿cuándo es excesivo el nivel de endeudamiento? Finanzas \& Desarrollo. Recuperado de: http://www.imf.org/external/pubs/ft/fandd/spa/2002/12/pdf/daseking.pdf

Dayton, J. (2008). Impuestos y gasto en América Latina: primero la estabilidad, ahora el desarrollo. Percepciones. Recuperado de: www.oecd.org/dev/percepciones Gómez, J. y Morán, D. (2013). Política tributaria en América Latina: agenda para una segunda generación de reformas. Macroeconomía del Desarrollo. Recuperado de: http://www.eclac.cl/publicaciones/xml/7/50237/S133MDD_Gomez_y_Moran.pdf Jiménez, J. y Andrea, P. (2009). Inversión, incentivos fiscales y gastos tributarios en América Latina. Macroeconomía del Desarrollo. Recuperado de: http://www.cepal.org/publicaciones/xml/2/35732/Serie_MD_77.pdf

López, G. (2010). La calidad del gasto público y su influencia en el desarrollo económico: una validación empírica para los países de la OCDE 19702005. Hacienda Pública Española. Revista de Economía Pública. Recuperado de:http://www. upf.edu/cres/en/publicacions/articles.html

Lora, E. (2006). El estado de las reformas del Estado en América Latina. Bogotá: Mayol Ediciones. Recuperado de: http://www.iadb.org/es/investigacion-y-datos/detalles-de-publicacion,3169.html?pub_id=b-616

Moodys. (27 de febrero de 2013). Perspectiva negativa en calificación de Honduras.

Recuperado de: http://www.centralamericadata.com/es/article/home/Moodys_Perspectiva_negativa_en_calificacin_de_Honduras.

Secretaría de Finanzas Públicas. (2014). Informe de liquidación presupuestaria. Recuperado de: http://www.sefin.gob.hn/?page_id=8246.

Secretaría de Finanzas Públicas. (2012). Datos de deuda pública interna y externa del Gobierno Central de Honduras del 2000 al 2012. Recuperado de: http://www.sefin.gob.hn/wp-content/uploads/2012/08/INFORME-DE-DEUDA -PUBLICA-DE-HONDURAS-II-Trimestre-2012.pdf 